\title{
Energy Dependence of the D-D Reaction Cross Section at Low Energies ${ }^{1}$
}

\author{
J. G. Brennan ${ }^{2}$ and J. J. Coyne
}

(August 11, 1964)

\begin{abstract}
The energy variation of the approach cross section for the D-D reaction is calculated using several different methods. The simplest method assumes strong absorption inside the nuclear surface and uses the WKB approximation. The slope of the Gamow plot is in this case a constant. A strong absorption model, without WKB approximation, leads to negative corrections to this constant slope. A weak absorption model, which seems more applicable, gives both positive and negative corrections, depending on the depth of the nuclear potential. Finally, since the amount of absorption seems important, the effects of varying the absorption are studied using the optical model. The possible influence of the $\mathrm{P}$-waves is also investigated. An experimental study of the energy dependence of the slope of the Gamow plot should give information on the optical potential which one deuteron sees when it approaches another deuteron.
\end{abstract}

\section{Introduction}

In an earlier treatment $[1]^{3}$ of the D-D reaction cross section, the strong energy dependence at low energies was attributed to the effects of the Coulomb and centrifugal barriers on the various angular momentum components of the incident beam. However, the barrier penetration factors were calculated using the WKB approximation for the Coulomb wave functions and retaining only the main energy dependence. If, in addition to these approximations, we also go to the low energy limit, then the energy dependence of the reaction cross section is given by the simple expression

$$
\sigma_{R}=(A / E) \exp \left(-B E^{-\frac{1}{2}}\right)
$$

where $A$ and $B$ are independent of the energy. A plot of the logarithm of $\sigma_{R} E$ as a function of $E^{-\frac{1}{2}}$ would give a straight line under these approximations. Such a plot is often called a Gamow plot and is frequently used in the analysis of the low energy data.

Looking at the experimental results from three groups [2-4] in the energy region of about 8 to 100 kev (center of mass), Brennan [5] has indicated that the data might suggest an increase in $B$ of eq (1) with energy. Brennan calculated the D-D reaction cross section using a low energy approximation [6] to the small absorption method of Ostrofsky, Breit, and Johnson [7]. In this present paper, the energy dependence of the reaction cross section has been studied under various assumptions suggested by this previous work.

\footnotetext{
1 Work supported by the National Seience Foundation and the Defense A tomic Support Agency.

2 The Catholic University of America, Washington, D.C

3 Figures in brackets indicate the literature references at the end of this paper.
}

Since retaining only the main energy dependence in the WKB approximation is equivalent to assuming the behavior of the wave function due to scattering from inside the nuclear surface does not affect the energy dependence of the cross section, the reaction cross section was first calculated using the continuum model [8], which assumes complete absorption inside the nucleus and, therefore, does not allow coherent scattering from inside the nuclear surface. The results of this calculation are compared with the results of reference 1. The approach used by Brennan, based on the small absorption model, involves an approximation valid only at low energies. To investigate the validity of this approximation, a second calculation was done, in which this approximation was not made.

Since these two calculations involved models assuming strong and weak absorption, the effects of absorption were studied in a third calculation, using the optical model [9]. An attempt was also made to study the influence of $\mathrm{P}$-waves on the cross section although only $\mathrm{S}$-waves were included in the three main calculations mentioned above.

\section{General Considerations}

In this paper, we are considering the $\mathrm{D}-\mathrm{D}$ reaction cross section at energies below $1 \mathrm{MeV}$ in the centerof-mass coordinate system. In this low energy region, it is valid to treat the initial kinetic energy as small compared with the energy of the nucleons once they are inside the nucleus. Since the energy dependence of the reaction cross section does not show any of the sharp peaks which are found when there is compound nuclear resonance, we can also assume that there are no resonances to compound nuclear states below $1 \mathrm{MeV}$.

Under these two assumptions, it is a good approximation to assume that the reaction cross section factors into two parts. The first part, the approach 
cross section, contains all of the energy dependence and measures the probability of the bombarding particle getting inside the target mucleus. The second part, the specific nuclear factor, is assumed to be independent of the energy and is related to the probability that, once the bombarding particle gets inside the target nucleus, the system will make a transition from the initial to the final state.

The reaction cross section can also be subdivided into cross sections for reactions initiated by particles with different $l$ values, i.e.,

$$
\sigma_{R}=\sum_{l}(4 / 9) P_{l} \sigma_{l}
$$

where $P_{l}$ is the specific nuclear factor for initial orbital angular momentum $l$ and $\sigma_{l}$ is the corresponding approach cross section. The factor $4 / 9$ arises from considerations of spin and symmetry [1]. Following previous treatments [1], we have ignored the contributions from the quintet spin states. In the present treatment, no attempt has been made to calculate the specific nuclear factors, $P_{l}$. They are assumed independent of the energy, and, therefore, do not affect the energy dependence of $\sigma_{R}$.

\section{WKB Approximation and the Continuum Model}

The continuum model [8] assumes complete absorption inside the nuclear surface and thus eliminates the effect of coherent scattering from inside the nucleus. The problem of calculating the approach cross section becomes one of calculating a potential barrier transmission coefficient. The approach cross section is related to the transmission coefficient as follows:

$$
\sigma_{l}=\pi \chi^{2}(2 l+1) T_{l}
$$

where $\pi \lambda^{2}(2 l+1)$ is the geometric limit and $T_{l}$ is the transmission coefficient. The transmission coefficients are given in terms of $\Delta_{l}$ and $S_{l}$, the penetration and shift factor, by Blatt and Weisskopf [10]. $\Delta_{l}$ and $S_{l}$ are, in turn, expressed in terms of the regular and irregular Coulomb wave functions and their derivatives [11].

If the WKB approximation is used for the Coulomb wave functions, and only the main energy dependence is retained, then we have [1]

$$
\begin{gathered}
\sigma_{l}=\exp \left[-2 C_{l}\right] \\
C_{l}=\int_{R}^{r_{l}}\left|k_{l}(r)\right| d r ; \\
k_{l}(r)=\left\{2 \mu / \hbar^{2}\left[E-e^{2} / r-\hbar^{2}\left(l+\frac{1}{2}\right)^{2} / 2 \mu r^{2}\right]\right\}^{\frac{1}{2}}
\end{gathered}
$$

and $r_{l}$ is the value of $r$ at which $k_{l}(r)=0$. It should be noted that in excluding all but the most important energy dependence, we have also eliminated all dependence on $U_{0}$, the depth of the nuclear potential.
The approach cross section, calculated using the WKB method, depends only on $R$, the nuclear radius, which we have chosen to be $7 \times 10^{-13} \mathrm{~cm}$ throughout this paper. This value of $R$ is the one commonly used in the D-D reaction calculations. Unless $R$ is changed drastically, the only effect of varying $R$ would be to change the height of the potential barrier.

Two calculations have been performed for the S-wave approach cross sections, one using the WKB approximation given by eq (4), the other using exact Coulomb wave functions [12] in the continuum model. In the second calculation, which we shall refer to simply as the continuum model, the dependence on $U_{0}$ was also retained. Figure 1 gives the slope of the Gamow plot for the WKB calculation and for the continuum model calculations with various well depths. In this figure and in all subsequent graphs of the slope of the Gamow plot, $E_{c}$ corresponds to the height of the Coulomb barrier, $205.69 \mathrm{keV}$; the scale marks $a$ and $b$ corresponds to $100 \mathrm{keV}$ and $8 \mathrm{keV}$, respectively. The depths of the nuclear potentials are given on the curves in $\mathrm{MeV}$. The results of figure 1 were obtained using numerical differentiation and using double interpolation of tabular data [12], good only to four significant

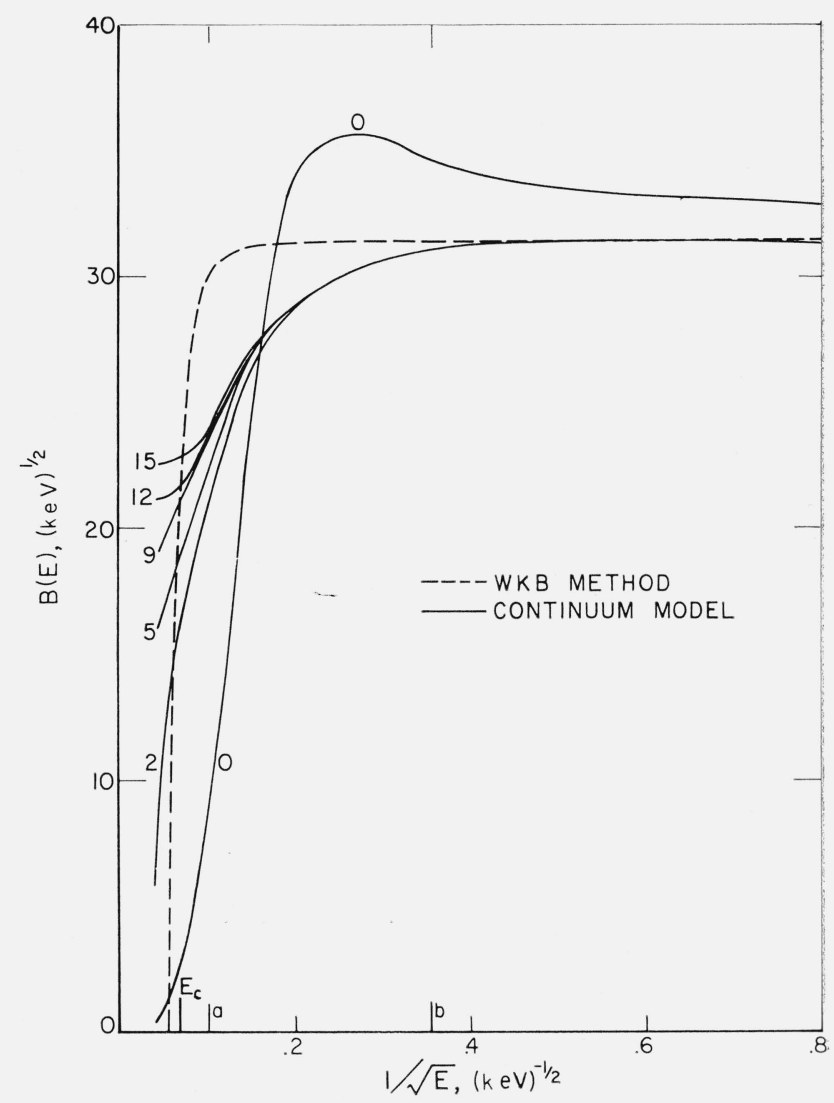

Figure 1. $\mathrm{B}(\mathrm{E})$ versus $\mathrm{E}^{-\frac{1}{2}}, W K B$ approximation and the continuum model.

In this and all subsequent figures, the numbers on the curves are the depths of the nuclear potential in $\mathrm{MeV}, \mathrm{E}_{\mathrm{c}}$ corresponds to the height of the Coulomb barrier, $a$ corresponds to $100 \mathrm{keV}$ and $b$ to $8 \mathrm{keV}$. 
figures. Consequently the accuracy of the curves shown is only about 10 percent. Except for $U_{0}=0$, which is certainly an unrealistic value for the depth of the nuclear potential, all of the continuum model curves give negative corrections to the WKB results.

In order to test the importance of higher angular momentum, the WKB calculations were performed also for $l=1,2$, and 3 . If we assume all $l$-waves have the same specific nuclear factors, then, for comparison, we can simply add the cross sections. Figure $2 b$ shows the effect of adding in higher and higher $l$ values, while figure 2a compares the slope of the Gamow plot for $\sigma_{0}$ with that for $\left(\sigma_{0}+\sigma_{1}\right)$. Higher $l$-waves were not included in figure 2 a because the results were not significantly different in the region of interest. While the addition of the P-waves can cause a positive correction to the slope of the Gamow plot, this correction is very small compared to the correction indicated by experiment. Moreover, in a later and a more accurate calculation involving $\mathrm{S}$ - and $\mathrm{P}$-waves, we shall see that the relative importance of higher $l$ values will depend very much on the depth of the nuclear potential.
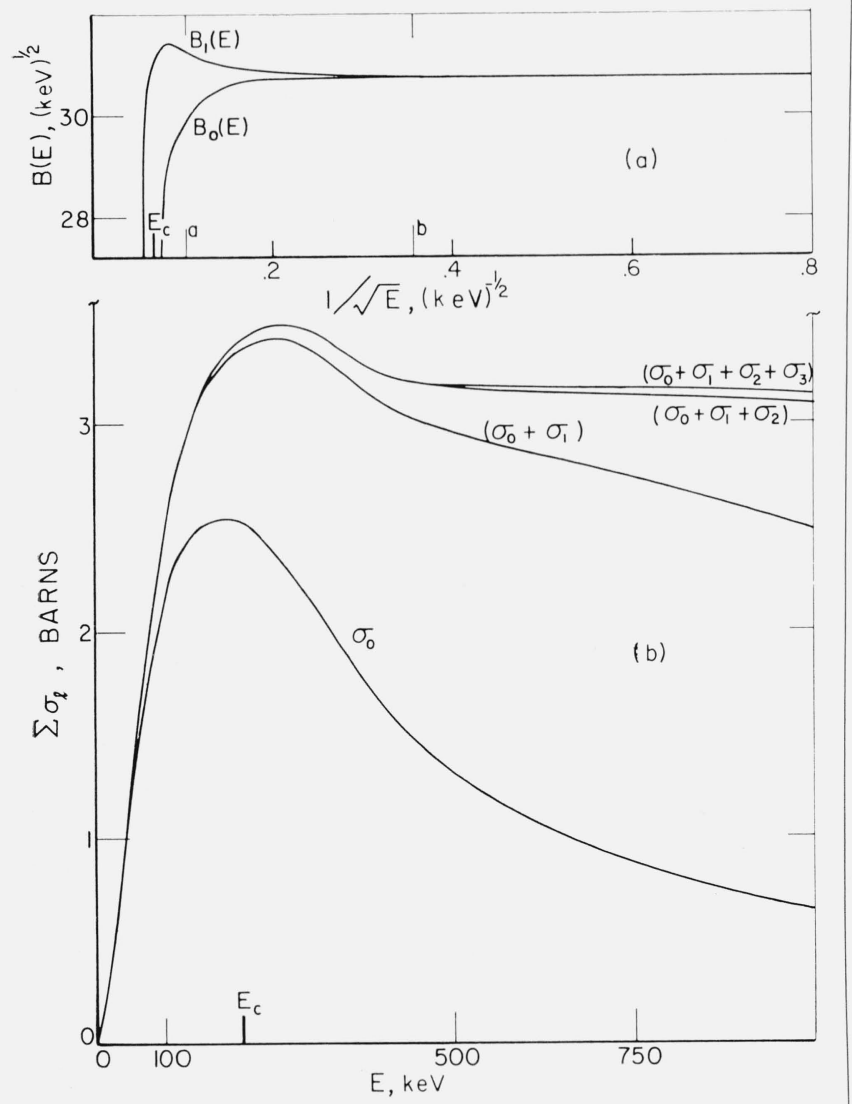

Figure 2a. $\mathrm{B}(\mathrm{E})$ versus $(\mathrm{E})^{-\frac{1}{2}}$ for $\mathrm{S}$ waves and for $(\mathrm{S}+\mathrm{P})$ waves in the WKB approximation.

FIGURE $2 \mathrm{~b} . \quad \Sigma \sigma_{l}$ versus $\mathrm{E}$ in the WKB approximation, showing the effects of higher $l$ waves.

\section{Small Absorption Approximation}

The treatment outlined in the previous section implied complete absorption inside the nucleus, since it ignored the effect of coherent scattering from inside the nucleus. The method described in this section assumes that the absorption is small, in fact so small that its effect on the probability density inside the nucleus can be ignored. This method was first introduced by Ostrofsky, Breit, and Johnson [7] and shall be referred to as the OBJ method. We will assume the absorption probability is the same throughout the nucleus, so that the shape factor for absorption will be one. This, we shall see later, is equivalent to choosing the imaginary part of the optical potential to be a square well. The approach cross section is the integral of the probability density over the nuclear volume divided by the flux of the incident beam. Since, in this method, the approach cross section is really a measure of the probability of being inside the nucleus, the specific nuclear factor is not a "branching ratio" but is related to the absolute probability of absorption.

The wave function inside the nucleus will be a solution for the nuclear potential which has been assumed. Outside the nucleus, the wave function is a linear combination of regular and irregular Coulomb wave functions, chosen so that the wave function and its derivative are continuous at the nuclear surface. With the wave function determined in this way, the approach cross section is given by eq (6) of Ostrofsky, Breit, and Johnson [7]. Using the OBJ method, we have treated only the S-waves, using a square well potential and also a clipped harmonic oscillator potential. The Coulomb wave functions for both calculations were the same as those used for the "continuum model" calculation. The square well calculation was carried out on a Burroughs E101 at the Georgetown College Observatory. The calculation for the harmonic oscillator was done on an IBM 704, located at the U.S. Naval Ordnance Laboratory.

Figure 3 gives Gamow plots for various depths of the nuclear potential. Besides the square well and the oscillator results, we have also included on each graph a plot of the WKB results normalized se that they correspond to the square well results at low energies and zero depth of the nuclear potential. The WKB results are included so that the variation of the curves with the depth of the nuclear potential will be more obvious.

While the results for a given depth may be quite different for the two potentials, the general behavior as a function of $U_{0}$ is the same. At a given depth the square well will give a curve of a certain shape, which can be duplicated by a harmonic oscillator well of greater depth. As we might suspect, the energy dependence of the low energy reaction cross section will not give us information of the shape of the nuclear potential, but will only indicate what depth must be chosen with a given shape, in order to get the same results. 


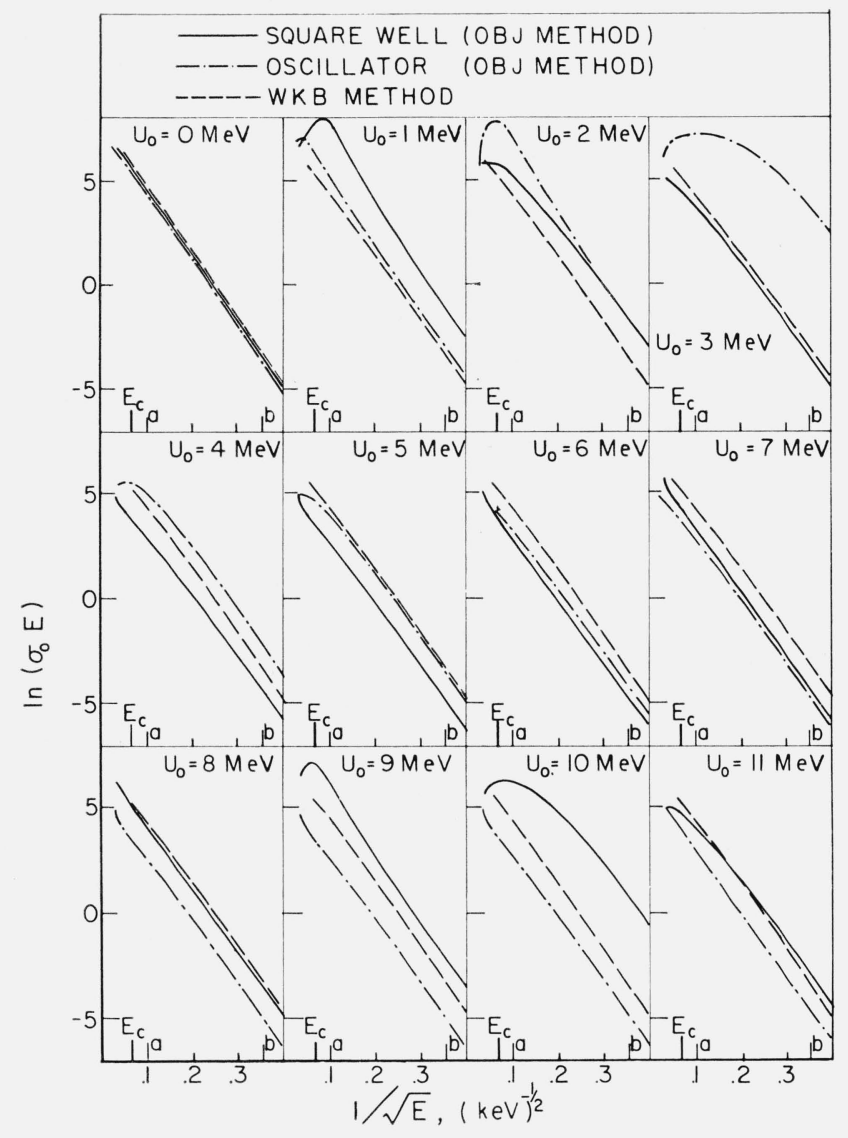

Figure 3. Gamow plots in the OBJ method for a square well and for a clipped harmonic oscillator nuclear potential, for various values for the depth, $\mathrm{U}_{0}$.

Figure 4 is a graph of the slope of the Gamow plot as a function of $E^{-\frac{1}{2}}$ for a square well of various depths. The WKB results are again included for comparison. This graph should be compared with figure 1 in order to compare the results of the "continuum" model and the OBJ method. The comments about the accuracy of the curves in figure 1 apply equally well to figure 4 . However, it is still clear that the OBJ method allows both positive and negative corrections to the WKB result, while the continuum model, for reasonable values of $U_{0}$, allows only a negative correction.

\section{Small Absorption Approximation at Very Low Energies}

Johnson and Jones [6] have shown that in the low energy limit the approach cross sections of the OBJ method are given by

$$
\sigma_{l}=\left(g_{l /} E\right)\left(e^{2 \pi \eta}-1\right)^{-1}\left(1+t_{l /} \eta^{2}\right)
$$

where $\eta=e^{2} / \hbar v \propto E^{-\frac{1}{2}}$ and $q_{l}$ and $t_{l}$ are energy in"dependent quantities, which are functions of the depth of the square well potential.

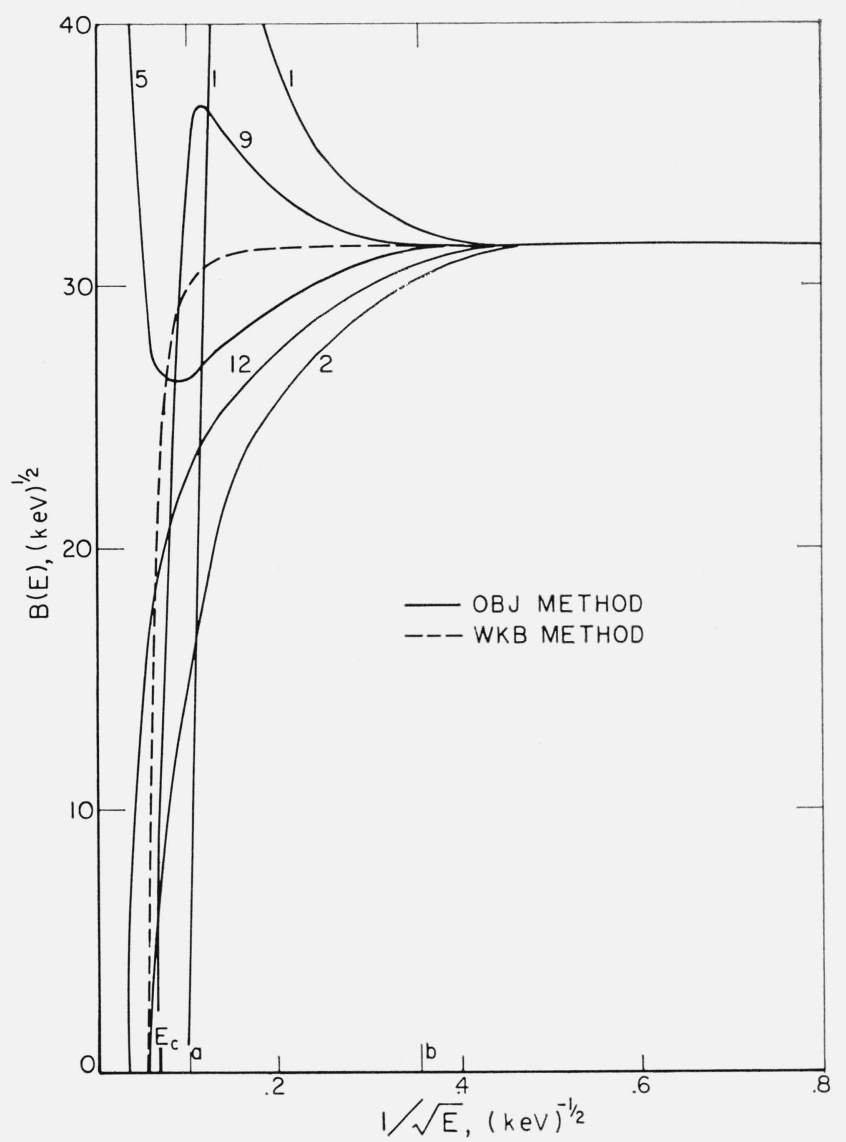

Figure 4. $\mathrm{B}(\mathrm{E})$ versus $\mathrm{E}^{-\frac{1}{2}}$ for a square well in the $O B J$ method.

The expression above was that applied to the D-D reaction cross section by Brennan [5]. He assumed that he was working at low enough energies that only the S-waves were important. If we consider only S-waves, the slope of the Gamow plot is given by

$$
d(\ln \sigma E) / d E^{-1 / 2}=-\left[(2 \mu)^{-1 / 2} 2 \pi \hbar / a\right]\left(1+t_{0} / \pi \eta^{3}\right)
$$

which corresponds to eq (5) of Brennan [5]. The right side of eq (6) is just the slope of the Gamow plot, $B$. The constant corresponds to the WKB result, while the second term includes a correction proportional to $E^{3 / 2}$. In figure 5 we plot $t_{0}$ as a function of $U_{0}^{\frac{1}{2}}$ (the other two curves will be referred to later). We see that the correction term can vary quite a bit depending on the value of $U_{0}$. Analyzing the available data [2-4], Brennan [5] decided on a value of $U_{0}$ in the vicinity of $9 \mathrm{MeV}$.

In order to show comparative results for the OBJ method, and the low energy limit, figure 6 gives the cross sections for both at different values of the depth. If we compare the values of $B$ calcualted, we find that, in general, the low energy limit (6) is valid. However, when $t_{0}$ is large, the results can differ considerably even at fairly low energies. 


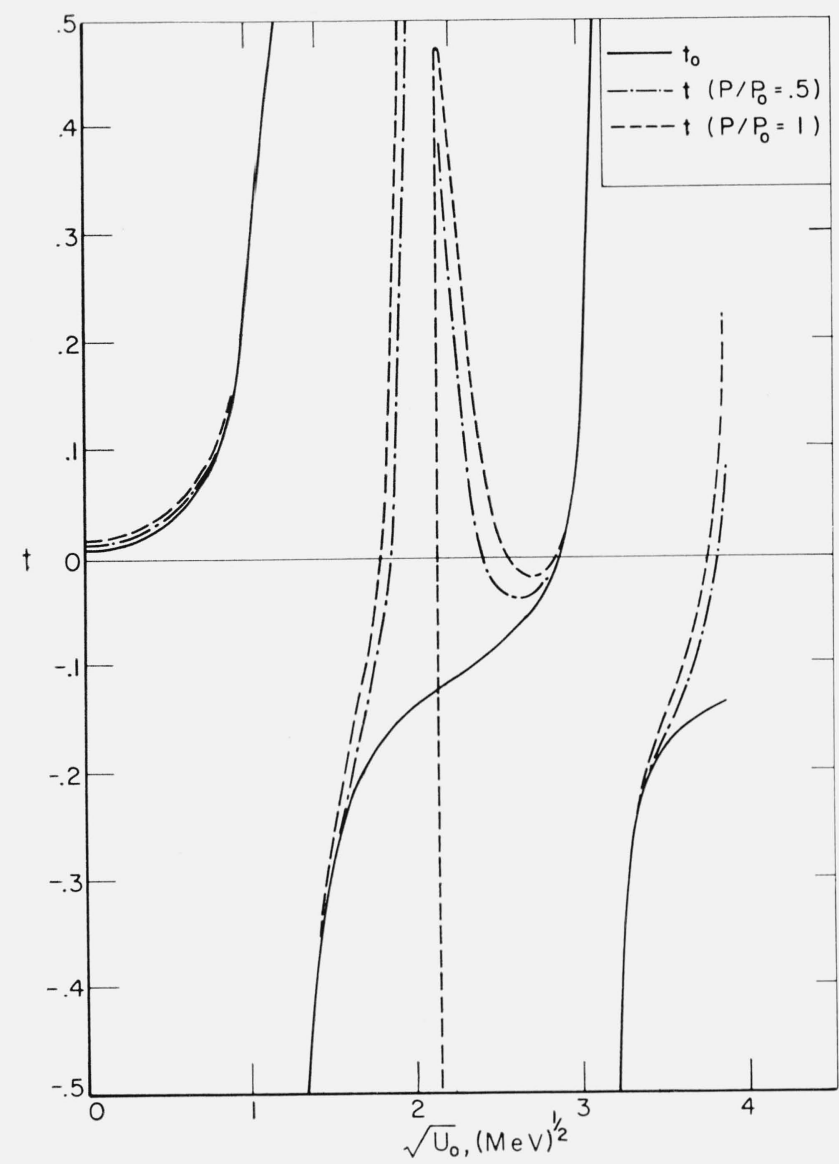

Figure 5. $\mathrm{t}_{0}$ and $\mathrm{t}$ of equations $(6)$ and $(8)$ versus $\mathrm{U}_{0^{\frac{1}{2}}}$.

In order to see how important the P-waves could be, the low energy limit (5) was also applied to the P-wave approach cross section. The results, however, are somewhat dependent on the ratio of the specific nuclear factors for the $\mathrm{S}$ - and $\mathrm{P}$-waves. Including the $\mathrm{P}$-waves, the total reaction cross section becomes

$$
\sigma_{R}=\frac{4 P_{0} q_{0}}{9 E\left(e^{2 \pi \eta}-1\right)}\left[1+\frac{t_{0}}{\eta^{2}}+\frac{P_{1} q_{1}}{P_{0}}\left(1+\frac{t_{1}}{\eta^{2}}\right)\right] .
$$

In terms of a Gamow plot this gives

$$
d(\ln \sigma E) / d E^{-\frac{1}{2}}=-\left[(2 \mu)^{-\frac{1}{2}} 2 \pi \hbar / a\right]\left(1+t / \pi \eta^{3}\right)
$$

where

$$
t=\frac{t_{0}+\left(P_{1} q_{1} / P_{0} q_{0}\right) t_{1}}{1+\left(P_{1} q_{1} / P_{0} q_{0}\right)}
$$

The quantity of interest is $t$ and as we see, it is a function of $P_{1} / P_{0}$. Figure 5 gives $t$ as a function of $U_{0}^{\frac{1}{2}}$ for $P_{1} / P_{0}=1.0$ and 0.5 . Comparing $t$ to $t_{0}$, we see that the $\mathrm{P}$-wave contribution can be very important or very unimportant, depending on the depth of the nuclear potential. In the vicinity of $9 \mathrm{MeV}$ the $\mathrm{P}$-wave is not very significant.

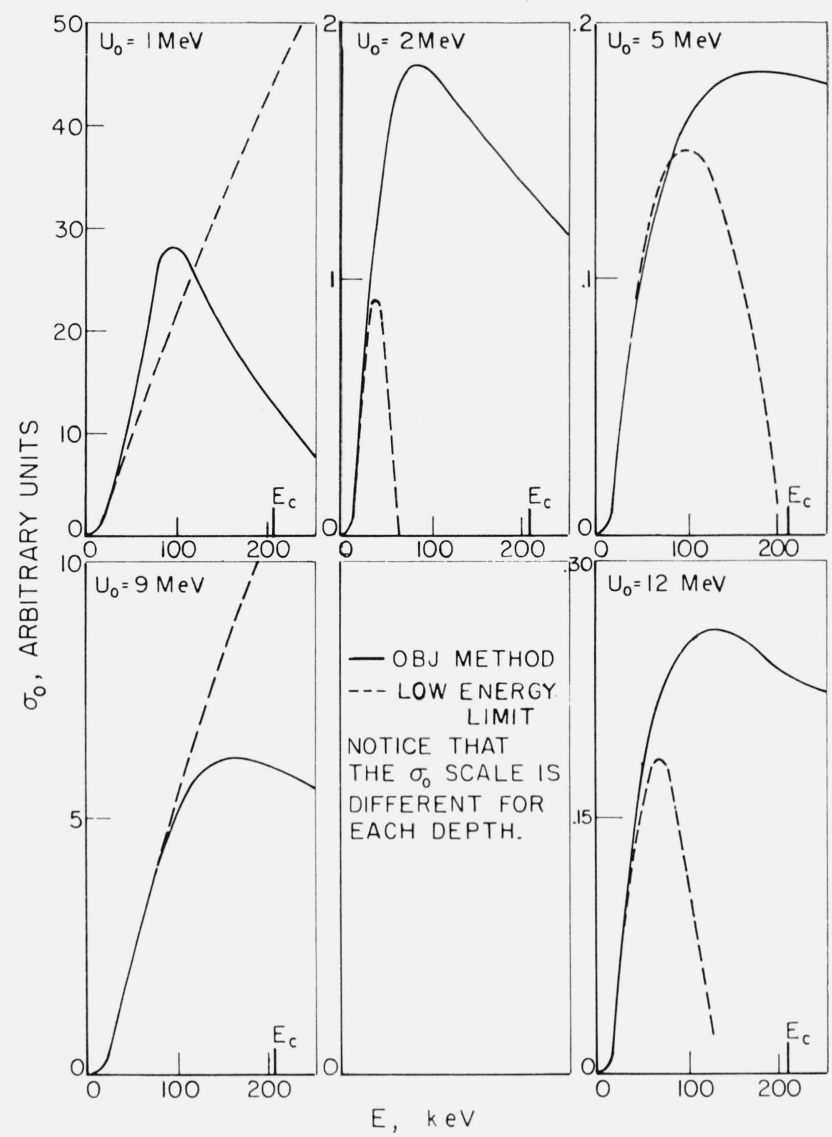

Figure 6. $\sigma_{0}$ versus E for the OBJ method and for the low energy limit, for various values of $\mathrm{U}_{0}$.

\section{Optical Model}

The methods for calculating the approach cross sections, which we have treated thus far, are at two extremes. Either we assume that the absorption inside the nucleus is complete or we assume that the absorption is so small that it is negligible compared to the scattering. In order to study the effect of varying the absorption, the S-wave approach cross sections were calculated, using the optical model [9]. The optical model leads to an absorption which is proportional to the probability density inside the nuclear surface. Since, as stated previously, $P_{0}$, the specific nuclear factor of the OBJ method, is proportional to the absolute probability of absorption, and $V_{c}$, the imaginary part of the optical potential, is proportional to the absorption in the optical model, the two methods should give the same results for small absorption. Comparing the reaction cross section for the optical model with that for the OBJ method, we find that, in the limit of small absorption,

$$
P_{0}=2 V_{c} / \pi \text {. }
$$




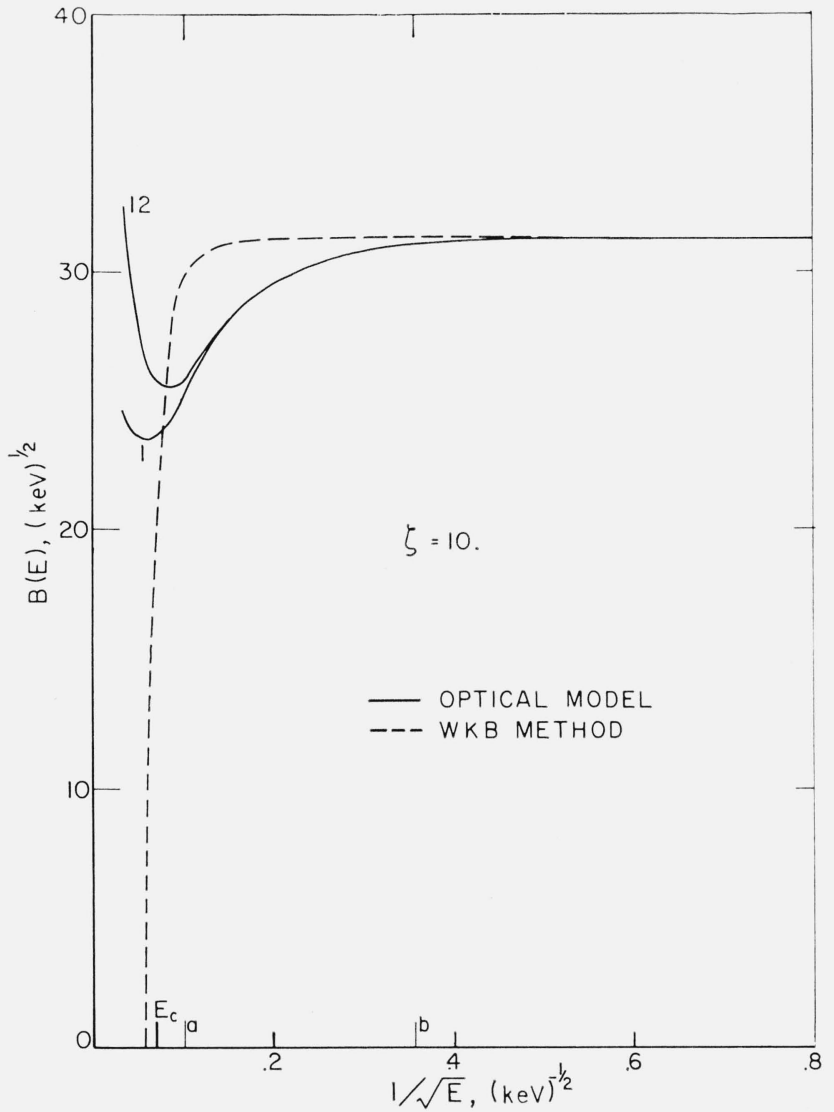

Figure 7. $\mathrm{B}(\mathrm{E})$ versus $(\mathrm{E})^{-\frac{1}{2}}$, the optical model. $\zeta$, the ratio of the imaginary to the real part of the potential $=10$.

Calculations in the optical model were carried out on the IBM 704 at the National Bureau of Standards. The potential used was a square well of the form $U_{0}(1+i \zeta)$. However, the Coulomb wave functions used were calculated directly by the computer and, therefore, are probably better than the Coulomb wave functions used in the previous calculations. S-wave approach cross sections were calculated for various values of $U_{0}$ and $\zeta$. Figures 7 through 11 give $B$, the slope of the Gamow plot as a function of $E^{-\frac{1}{2}}$. Comparing figure 11 with figure 4 , we see that the results for $\zeta=0.0001$ and the OBJ method compare as favorably as we can expect. Calculations were also done for $\zeta=0.0002$, but the cross section results differed from $\zeta=0.0001$ results by only a factor of 2 , while the $B$ results coincided in all cases. This indicates that when the absorption is low enough to make $\zeta=0.0001$, the OBJ method is surely a good approximation, i.e., the absorption does not affect the approach cross section.

There is no continuum model equivalent in the optical model, for as the imaginary part of the potential increases, the absorption inside the nucleus increases, but the reflection at the nuclear surface also increases. As the imaginary part of the optical potential approaches very large values the reaction cross section goes to zero. However, a comparison of figures 1 and 7 shows that the shape of the $B$ versus $E^{-\frac{1}{2}}$ curves is roughly the same for the continum model and the optical model with large values for $\zeta$.

\section{Summary and Conclusions}

The experimental results for the $\mathrm{D}-\mathrm{D}$ reaction cross section at low energies are usually given in terms of a straight line on a Gamow plot. Using the WKB method, we have found that the calculated value for $B(E)$, the slope of the Gamow plot, is indeed very close to constant in the energy region from 8 to $100 \mathrm{keV}$. The "continuum" model, which is the more accurate strong absorption approximation, gives a negative energy dependent correction to the constant value predicted for $B(E)$ by the WKB method. Brennan [5] has pointed out that the experimental results seem to indicate a positive correction to $B(E)$. In addition, theoretical considerations seem to indicate that a weak, rather than

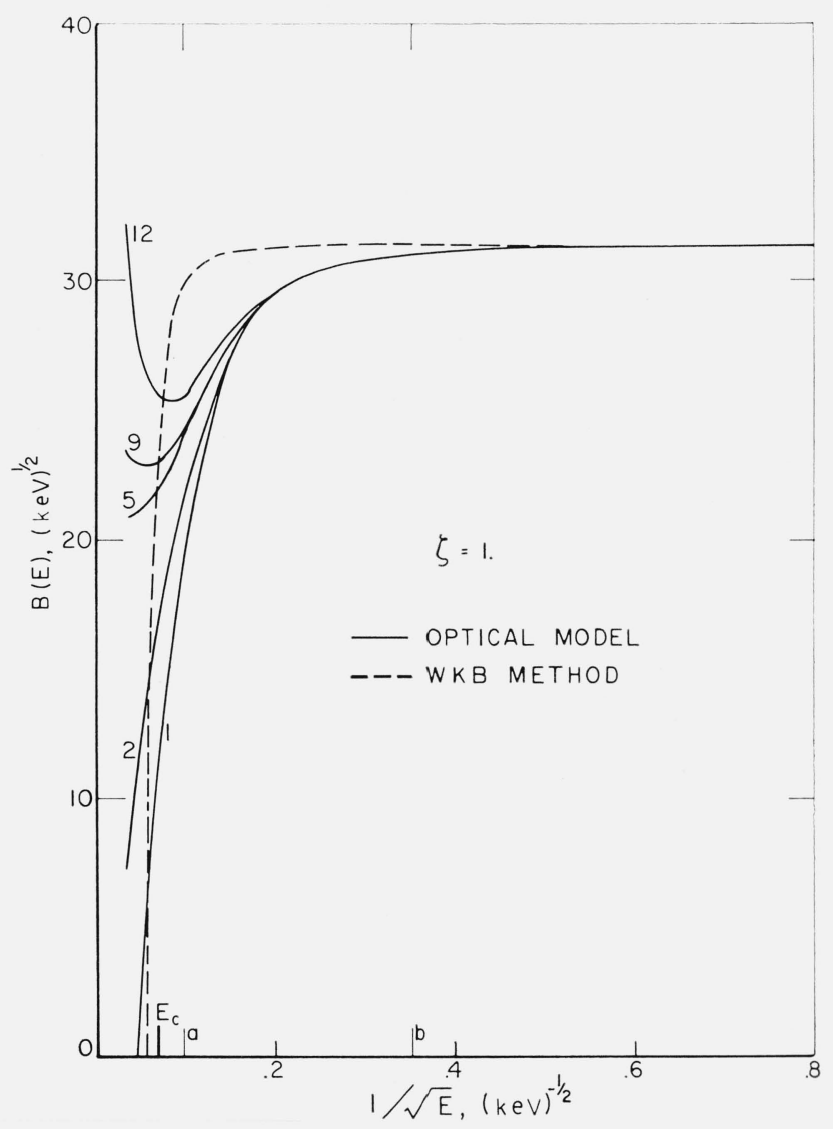

Figure 8. $\mathrm{B}(\mathrm{E})$ versus $\mathrm{E}^{-\frac{1}{2}}$, optical model, $\zeta=1$. 


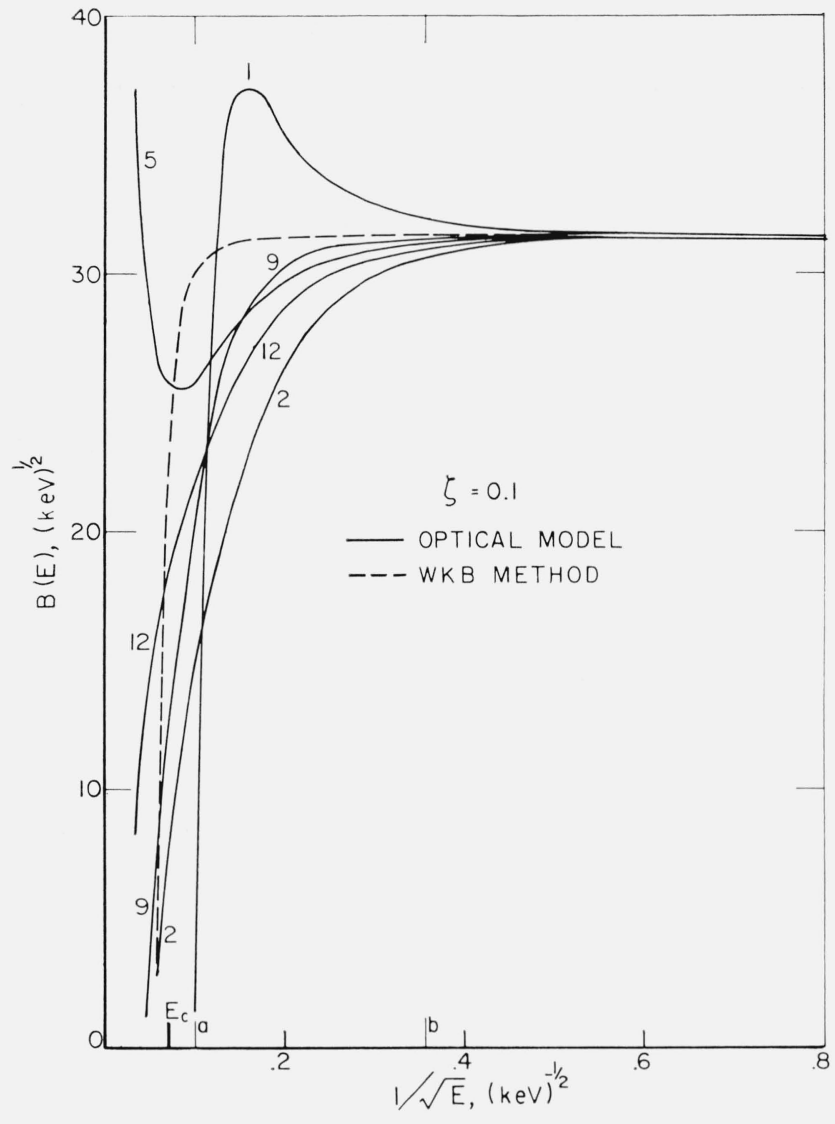

Figure 9. $\mathrm{B}(\mathrm{E})$ versus $\mathrm{E}^{-\frac{1}{2}}$, optical model, $\zeta=10^{-1}$.

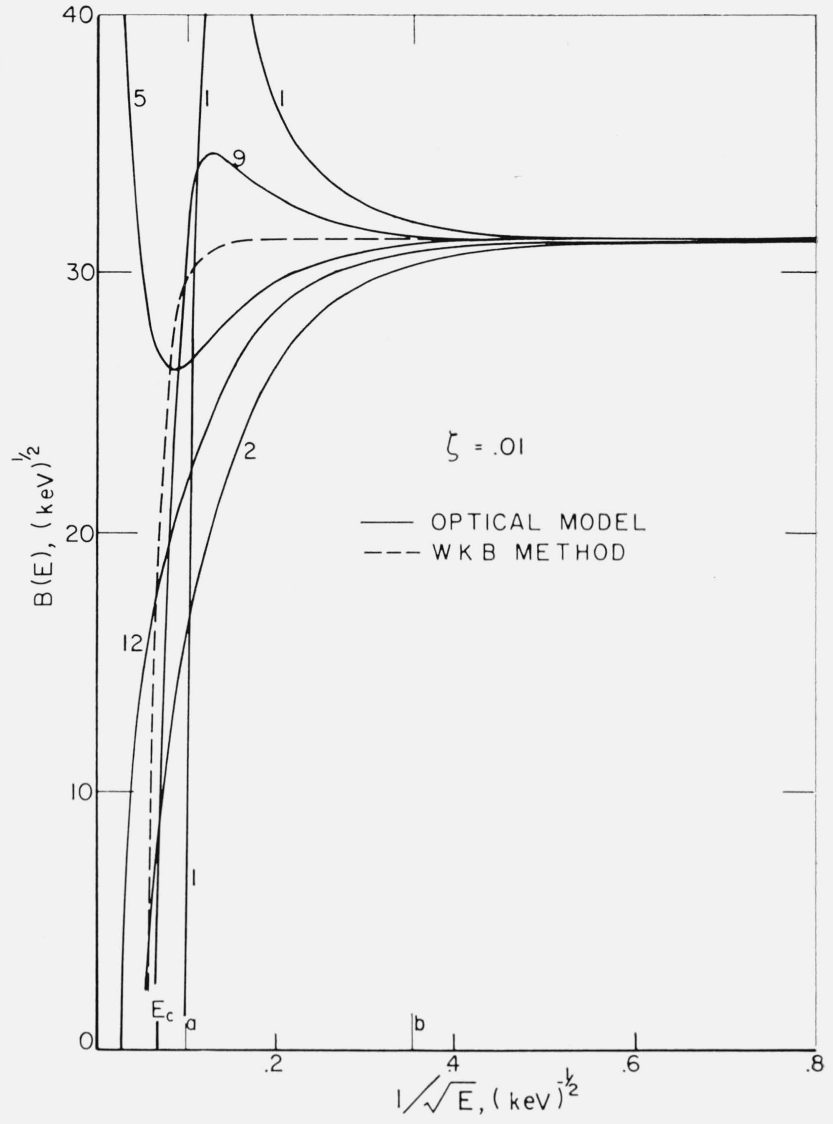

Figure 10. $\mathrm{B}(\mathrm{E})$ versus $\mathrm{E}^{-\frac{1}{2}}$, optical model, $\zeta=10^{-2}$.

that the importance of the P-waves was very much a function of the depth chosen for the potential. More accurate experimental results for the total reaction cross section will, we hope, lead to a better knowledge of the energy dependence of $B(E)$, which in turn would tell us more about the optical potential proper to the collision between two deuterons. Also, more accurate studies of the energy dependence of the angular distribution of the reaction products might be used to investigate the influence and importance of the P-waves.

The authors express their gratitude to the U.S. Naval Ordnance Laboratory, White Oak, Silver Spring, Md., to the Georgetown College Observatory, Washington, D.C., and to the National Bureau of Standards, Washington, D.C., for the generous use of their computer facilities. 


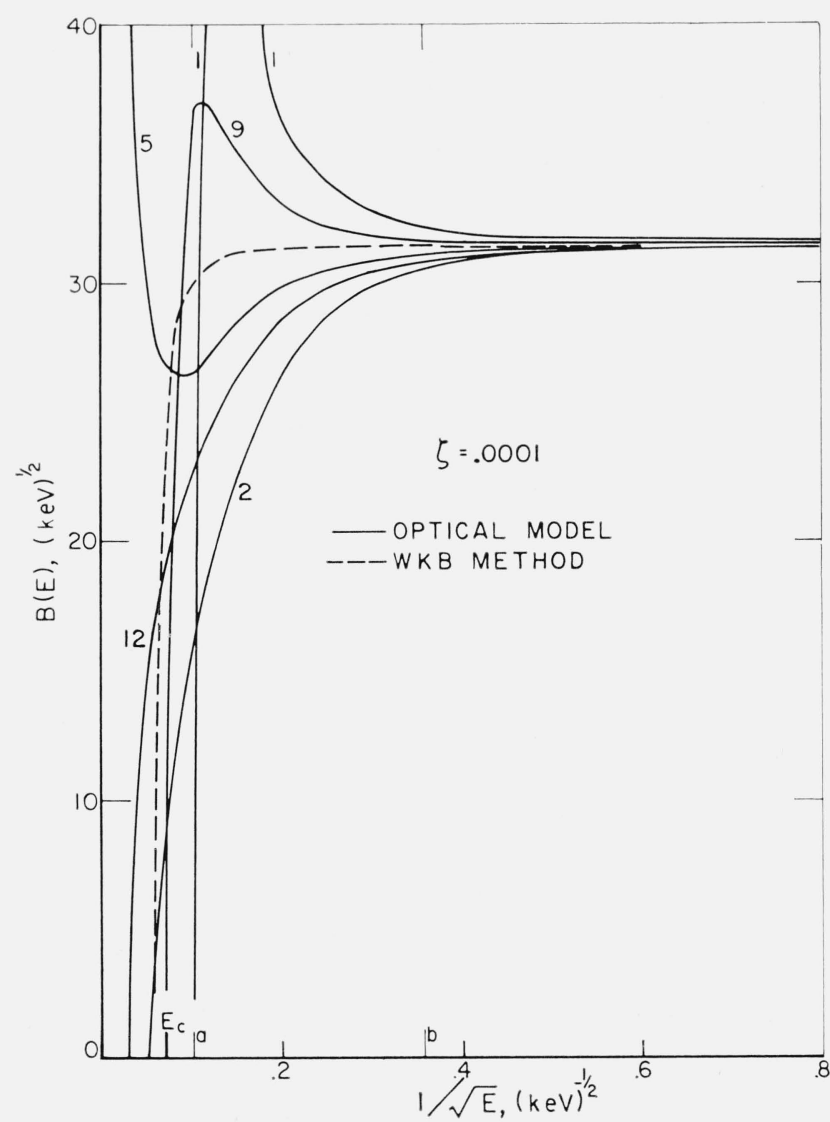

Figure 11. $\mathrm{B}(\mathrm{E})$ versus $\mathrm{E}^{-\frac{1}{2}}$, optical model, $\zeta=10^{-4}$.

\section{References}

[1] F. M. Beiduk, J. R. Pruett, and E. J. Konopinski, Phys. Rev. 7\%, 622 (1950).

[2] Arnold, Phillips, Sawyer, Stovall, and Tuck, Phys. Rev. 93, 483 (1954).

[3] Eliot, Roaf, and Shaw, Proc. Roy. Soc. (London) A216, 57 (1953).

[4] Davenport, Jeffries, Owen, Price, and Roaf, Proc. Roy. Soc. (London) A216, 66 (1953).

[5] J. G. Brennan, Phys. Rev. 111, 1592 (1958).

[6] J. L. Johnson and H. M. Jones, Phys. Rev. 93, 1286 (1954).

[7] M. Ostrofsky, G. Breit, and D. P. Johnson, Phys. Rev. 49, 22 (1936).

[8] H. Feshbach, D. C. Peaslee, and V. F. Weisskopf, Phys. Rev. 71, 145 (1947).

[9] H. Feshbach, C. E. Porter, and V. F. Weisskopf, Phys. Rev. 96, 448 (1954).

[10] J. M. Blatt and V. F. Weisskopf, Theoretical Nuclear Physics, p. 360 (John Wiley and Sons, Inc., New York, N.Y., 1957).

[11] Ibid., p. 332

[12] I. Bloch, M. M. Hull, A. A. Broyles, W. G. Bouricius, B. E. Freeman, and G. Breit, Rev. Mod. Phys. 23, 147 (1951). 